ORIGINAL ARTICLE

\title{
Biofilm formation and esp gene carriage in enterococci
}

\author{
A A Ramadhan, E Hegedus
}

J Clin Pathol 2005;58:685-686. doi: 10.1136/icp.2004.024109

\begin{abstract}
Aims: To investigate biofilm production and esp carriage in enterococci.
Methods: Biofilm production in vancomycin resistant enterococci (VRE) and vancomycin susceptible enterococci (VSE) was tested on a microtitre plate method, using both brain heart infusion (BHI) broth and human serum as media. Isolates were screened for the esp gene, which has been reported to be essential for biofilm formation in enterococci, by means of the polymerase chain reaction.

Results: None of seven VRE and nine of 28 VSE tested formed a biofilm. One initially negative VRE Enterococcus faecium isolate produced a strong biofilm after 21 weeks of dry starvation on a cotton swab. By Fisher's exact test, there was no significant difference in biofilm formation between VRE and VSE, $E$ faecalis and $E$ faecium, or isolates from different sites. Biofilm formation was independent of possession of the esp gene. One isolate produced a strong biofilm in human serum but only a weak biofilm in BHI, whereas another produced a moderate biofilm in human serum but a weak biofilm in BHI.

Conclusions: The acquisition of vancomycin resistance may result in a lower ability to form biofilms, but a larger study using clinical isolates is needed to test this hypothesis. That one initially negative VRE isolate produced a strong biofilm after prolonged dry starvation suggests that biofilm formation may be an adaptive response. The esp gene does not appear to be necessary or sufficient for production of biofilms in enterococci.
\end{abstract}

See end of article for authors' affiliations

Correspondence to: Mr A A Ramadhan, School of Biomedical Sciences, Faculty of Health, Sciences, Cumberland Campus, PO Box 170, Lidcombe, NSW 1825, Australia; Abdull20@yahoo.com

Accepted for publication 9 November 2004

A biofilm is an assemblage of microbial cells that is irreversibly associated with a surface and enclosed in a matrix of primarily polysaccharide material. ${ }^{1}$ Biofilms are important both in nature, where they can enhance survival under unfavourable circumstances, and in medicine. Biofilms formed in medical devices, both inside and outside the body, can promote and sustain infections. Because of the restricted penetration of antimicrobials, decreased growth rate, and expression of possible resistance genes, they are not easily eradicated by bactericidal antibiotics. ${ }^{2-4}$ Biofilm forming Enterococcus faecalis have also been shown to survive better in macrophages than negative isolates. ${ }^{5}$

Shankar et al reported the identification of a new cell wall associated protein of $E$ faecalis, Esp, which was enriched in infection derived isolates. ${ }^{6}$

"Biofilms are important both in nature, where they can enhance survival under unfavourable circumstances, and in medicine"

Toledo-Arana et al reported that (1) the capacity to form biofilms is common among clinical $E$ faecalis isolates; (2) biofilm formation capacity is restricted to strains harbouring the esp gene; and (3) Esp promotes primary attachment of and biofilm formation by $E$ faecalis on abiotic surfaces. ${ }^{7}$

However, Dworniczek et al and Mohamed et al found that the esp gene was not required for biofilm formation. ${ }^{8} 9$

Another recent study demonstrated the formation in vitro of biofilms by E faecalis in the absence of, not only the esp gene, but also the entire pathogenicity island harbouring the esp coding sequence. ${ }^{10}$

Our study set out to determine the ability of vancomycin susceptible enterococci (VSE) and vancomycin resistant enterococci (VSE) from various sources to form biofilms, and any correlation between biofilm formation and the presence of the esp gene.

\section{METHODS}

Phosphate buffered saline washed cells from a brain heart infusion (BHI) broth culture of enterococcus isolates were used in a microtitre plate assay for biofilm production, using the method described by Djordjevic et al. ${ }^{11}$ VRE cultures were also tested for biofilm formation after assay for the maximum time of survival on dry cotton swabs at room temperature.

All strains of enterococci included in the study were screened for the esp gene by the polymerase chain reaction (PCR), using primers described previously. ${ }^{12}$ The reaction mixtures consisted of the following: $25 \mu \mathrm{l}$ PCR master mix (Qiagen, Hilden, Germany), $5 \mu \mathrm{l}$ primer $(2 \mu \mathrm{M}), 15 \mu \mathrm{l}$ sterile water, and $5 \mu \mathrm{l}$ DNA. PCR was carried out with the following thermal cycling profile: initial activation step at $95^{\circ} \mathrm{C}$ for 15 minutes; 30 cycles of denaturation at $90^{\circ} \mathrm{C}$ for 30 seconds, annealing at $58^{\circ} \mathrm{C}$ for one minute, and extension at $72^{\circ} \mathrm{C}$ for one minute; final extension at $72^{\circ} \mathrm{C}$ for 10 minutes.

\section{RESULTS}

Table 1 shows the results.

None of the VRE isolates tested initially formed a biofilm, compared with nine of the VSE isolates tested. However, this difference was not significant by Fisher's exact test $(p=0.153)$. One of the VRE isolates produced a strong biofilm after 21 weeks of dry starvation.

Three of 11 vancomycin susceptible $E$ faecium isolates and six of 17 vancomycin susceptible $E$ faecalis isolates showed biofilm formation. Again, the difference was not significant by Fisher's exact test $(p=1)$.

The proportions of biofilm forming VSE isolates taken from different sites varied: two of four for blood, three of six for wound swabs, one of three for sterile body cavity, and three of 13 for urine. These differences were not significant.

The three biofilm forming urinary isolates were the only ones to produce a strong biofilm in both test media. One $E$ faecalis isolate from blood produced a strong biofilm in serum but only a weak biofilm in BHI, whereas an $E$ faecalis wound isolate produced a moderate biofilm in serum but a weak biofilm in BHI.

Abbreviations: $\mathrm{BHI}$, brain heart infusion; $\mathrm{PCR}$, polymerase chain reaction; VRE, vancomycin resistant enterococci; VSE, vancomycin susceptible enterococci 
Table 1 Biofilm formation and esp gene in enterococcal isolates

\begin{tabular}{lllll}
\hline $\begin{array}{l}\text { Enterococcus } \\
\text { species }\end{array}$ & $\begin{array}{l}\text { Biofilm+ } \\
\text { /esp+ }\end{array}$ & $\begin{array}{l}\text { Biofilm+ } \\
\text { /esp- }\end{array}$ & $\begin{array}{l}\text { Biofilm- } \\
\text { /esp+ }\end{array}$ & $\begin{array}{l}\text { Biofilm- } \\
\text { /esp- }\end{array}$ \\
\hline VR E faecium & 0 & 0 & 0 & 5 \\
VR E faecalis & 0 & 0 & 0 & 2 \\
VS E faecium & 2 & 1 & 3 & 6 \\
VS E faecalis & 5 & 1 & 0 & 8 \\
\hline
\end{tabular}

VR, vancomycin resistant; VS, vancomycin susceptible.

Seven biofilm producing isolates were esp positive, whereas two were negative. Three non-biofilm producing isolates were esp positive.

\section{DISCUSSION}

We found an apparently almost random distribution of the ability to form biofilms among VSE hospital isolates. A weakness of this analysis was that all the isolates were obtained from a single hospital. An even more serious weakness was the inability to obtain clinical data; some of the isolates may have been colonising rather than infecting. A study with a large number of isolates from different sites and with clinical data enabling at least an informed guess as to whether the isolate was or was not definitely pathogenic at the site would be of interest. Another facet that could be examined is whether the isolate was associated with the use of equipment favouring biofilm formation (such as catheters); a correlation between enterococcal biofilm formation and catheter related bloodstream infections has been demonstrated. ${ }^{13}$

"Another facet that could be examined is whether the isolate was associated with the use of equipment favouring biofilm formation (such as catheters)"

The general lack of biofilm formation by VRE shown in our study may have resulted from the fact that these organisms were colonisers, and clinical isolates would need to be examined before the possibility raised in our study-that acquisition of vancomycin resistance results in a lower ability to form biofilms-could be confirmed.

That one esp negative VRE isolate, initially negative for biofilm formation, produced a strong biofilm after prolonged dry starvation suggests that biofilm formation may be an adaptive response.

The results indicate that possession of the esp gene is neither necessary nor sufficient for production of biofilms in enterococci.

Human serum may be a more sensitive medium than BHI broth for demonstrating biofilm formation in enterococci.
Take home messages

- The acquisition of vancomycin resistance may result in a lower ability to form biofilms, but a larger study using clinical isolates is needed to test this hypothesis

- Biofilm formation may be an adaptive response

- Possession of the esp gene does not appear to be necessary or sufficient for production of biofilms in enterococci

- Human serum may be a more sensitive medium than brain heart infusion broth for demonstrating biofilm formation in enterococci

\section{Authors' affiliations}

A A Ramadhan, E Hegedus, School of Biomedical Sciences, Faculty of Health, Sciences, Cumberland Campus, PO Box 170, Lidcombe, NSW 1825, Australia

\section{REFERENCES}

1 Donlan RM. Biofilms: microbial life on surfaces. Emerg Infect Dis 2002;8:881-90.

2 Costerton JW, Stewart PS, Greenberg EP. Bacterial biofilms: a common cause of persistent infections. Science 1999;284:1318-22.

3 Gilbert P, Das J, Foley I. Biofilm susceptibility to antimicrobials. Adv Dent Res 1997;11:160-7.

4 Maira-Litran T, Allison DG, Gilbert P. An evaluation of the potential of the multiple antibiotic resistance operon (mar) and the multidrug efflux pump arcAB to moderate resistance towards ciprofloxacin in Escherichia coli biofilms. J Antimicrob Chemother 2000;45:789-95.

5 Baldassarri L, Bertuccini L, Ammendolia MG et al. Receptor-mediated endocytosis of biofilm-forming Enterococcus faecalis by rat peritoneal macrophages. Indian J Med Res 2004;119:131-5.

6 Shankar V, Baghdayan AS, Huycke MM, et al. Infection-derived Enterococcus faecalis strains are enriched in esp, a gene encoding a novel surface protein. Infect Immun 1999;67:193-200.

7 Toledo-Arana A, Valle J, Solano C, et al. The enterococcal surface protein, Esp, is involved in Enterococcus faecalis biofilm formation. Appl Environ Microbiol 2001;67:4538-45.

8 Dworniczek R, Kuzko K, Mroz E, et al. Virulence factors and in vitro adherence of Enterococcus strains to urinary catheters. Folia Microbiol (Praha) 2003:48:671-8.

9 Mohamed JA, Huang W, Nallapareddy SR, et al. Influence of origin of isolates, especially endocarditis isolates, and various genes on biofilm formation by Enterococcus faecalis. Infect Immun 2004;72:3568-63.

10 Kristich CJ, Li YH, Cvitkovitch DG, et al. Esp-independent biofilm formation by Enterococcus faecalis. J Bacteriol 2004;186:154-63.

11 Diordjevic D, Wiedmann M, McLandsborough LA. Microtiter plate assay for assessment of Listeria monocytogenes biofilm formation. Appl Environ Microbiol 2002;68:2950-8.

12 Woodford N, Soltani M, Hardy KJ. Frequency of esp in Enterococcus faecium isolates. Lancet 2001;358:584.

13 Sandoe JAT, Witherden IR, Cove JH, et al. Correlation between enterococcal biofilm formation in vitro and medical-device-related infection potential in vivo. J Med Microbiol 2003;52:547-50. 